\title{
Development of the Cherenkov-type diagnostic system to study runaway electrons within tokamaks
}

\author{
Lech Jakubowski, Karol Malinowski, Robert Mirowski, Marek Rabiński', \\ Marek J. Sadowski, Jarosław Żebrowski and Marcin J. Jakubowski \\ National Centre for Nuclear Research (NCBJ) \\ 7 Andrzeja Soltana Str., 05-400 Otwock, Poland \\ E-mail: marek.rabinski@ncbj.gov.pl
}

\begin{abstract}
Measurements of fast electrons produced in plasma and escaping from tokamak-type facilities are of particular interest for diagnostics, because such electrons deliver information about processes occurring inside bulk plasma. A team from the National Centre for Nuclear Research (NCBJ, former IPJ) proposed Cherenkov-type probes for measurements of fast electrons in tokamaks because of high spatial- and temporal-resolution of such detectors.

The paper presents feasibility and design studies of the Cherenkov-type probes, a development of the measuring head constructions designed for different tokamak devices. In order to lower an energy threshold of the electron detection the authors applied radiators with the highest values of the refractive index. Different radiator materials, such as aluminium nitride (AlN) and CVD diamond crystals were applied. Several versions of measuring heads and different manipulators, e.g., a movable vacuum-tight shaft or a fast-moving reciprocating probe, have been manufactured and used. The practical application of the Cherenkov probes required also a consideration of the optical fibres and photomultipliers spectral characteristics. In order to investigate an energy distribution of fast electron beams the multi-channel probes have been designed and manufactured.
\end{abstract}

First EPs Conference on Plasma Diagnostics - $1^{\text {st }}$ ECPD

14-17 April 2015,

Villa Mondragone, Frascati (Rome) Italy

${ }^{1}$ Speaker 


\section{Introduction}

The Cherenkov radiation is emitted by a charged particle moving through a transparent medium with a velocity higher than the phase velocity of light in this medium. Emitted energy slowly increases with an increase in the particle velocity and it is larger for a medium with a larger refraction coefficient. From a comparison of refraction index values and corresponding minimal energy thresholds for different materials one could conclude that the use of radiators made of diamond or rutil crystals enables to record electron beams of lower energy.

Studies of pulsed streams of fast electrons in various experimental facilities of the PlasmaFocus type and so-called Rod Plasma Injectors have been carried out by means of various Cherenkov-type detectors by the NCBJ (former IPJ) team for many years [1]. Taking into account advantages of this diagnostic techniques it was decided to use the accumulated experience for measurements of fast electrons also in tokamaks [2].

Experimental studies of the fast electron beams have been performed within several tokamaks, namely in the CASTOR (in Prague), TORE SUPRA (in Cadarache), ISTTOK (in Lisbon), COMPASS (in Prague) and FTU (in Frascati) [3-4]. The obtained experimental results demonstrated that relatively intense Cherenkov signals appear particularly during the final phase of the discharge, when the tokamak plasma column is disrupted.

\section{Measuring head design}

The practical realization of the Cherenkov-type diagnostics induces some serious problems. The main design problem is to ensure an effective heat transfer through the radiator and a shielding body, the formation of an appropriate heat sink, as well as keeping temperatures of the radiator and its shield below the maximum admissible values. The electron streams, e.g. ripple-born electrons within the TORE SUPRA facility, were produced during the additional Low Hybrid plasma heating. At such conditions the fast electrons and ions could drift along the izo-B surface deeply inside the volumes of the vertical diagnostic ports. An energy spectrum of such electrons ranges from about $50 \mathrm{keV}$ to above $300 \mathrm{keV}$, and the electron current density amounts to about $20 \mathrm{~A} / \mathrm{m}^{2}$. A pulsed stream of such electrons can bombard the internal surface of the tokamak vacuum chamber and deposit about $5 \mathrm{MW} / \mathrm{m}^{2}$ of heat load, what in turn can cause local destructions of the chamber wall material, as well as a damage of the measuring head.

In general, the electron-induced intense heat loads require the use of materials resistant to high temperature, the application of materials with a high thermal conductivity, and the performance of all measurements during a relatively short time. Table 1 presents the values of retractive indexes, corresponding electron energy thresholds and thermal parameters of applicable materials.

On the basis of a comparative analysis of the thermal and optical properties, the threshold values presented above, as well as other factors, it was decided to apply the radiator made of a diamond crystal, which has an excellent thermal conductivity (four times higher than that of copper) enabling to dissipate heat deposited upon the radiator surface, mainly in a layer of a few hundreds micrometers in the depth. The diamond's refractive index is high enough to observe the Cherenkov radiation from electrons of energy higher than about $50 \mathrm{keV}$, as shown in Table 1. 
Table 1. Parameters of different materials applicable as the Cherenkov radiators.

\begin{tabular}{|l|c|c|c|}
\hline \multicolumn{1}{|c|}{ Material } & $\begin{array}{c}\text { Refraction } \\
\text { index }\end{array}$ & $\begin{array}{c}\text { Lower electron } \\
\text { energy threshold } \\
{[\mathrm{keV}]}\end{array}$ & $\begin{array}{c}\text { Thermal conductivity } \\
{\left[\mathrm{W} \mathrm{m}^{-1} \mathrm{~K}^{-1}\right]}\end{array}$ \\
\hline Glass & 1.80 & 104 & 1 \\
Aluminium nitride (AlN) & 2.15 & 66 & 170 \\
Diamond & 2.42 & 51 & 2000 \\
Rutile $\left(\mathrm{TiO}_{2}\right)$ & 2.90 & 33 & 12 \\
\hline
\end{tabular}

In the past, in some applications the use was also made of aluminium nitride (AlN) radiators. In the case of the Cherenkov head for multi-channel measurements it was decided to split the whole electron energy spectrum into channels with different lower energy thresholds determined by a thickness of the applied absorption foil-filter. As the absorption filter material was chosen pure molybdenum (Mo), due to its good thermal diffusivity and resistance to the sputtering. The chosen values of the filter thickness and corresponding energy threshold values for aluminium-nitride and diamond radiators are given in Table 2.

Table 2. Minimum energy of electrons, which could be recorded by AlN and diamond radiators covered by the chosen Mo-foil filters $\left(\rho=10.22 \mathrm{~g} \mathrm{~cm}^{-3}\right)$.

\begin{tabular}{|c|c|}
\hline \multicolumn{2}{|c|}{ Aluminium nitride radiator [3] } \\
\hline Thickness of the Mo-foil filter & Minimum energy of the recorded electrons \\
\hline $1 \mu \mathrm{m}$ & $69 \mathrm{keV}$ \\
$3 \mu \mathrm{m}$ & $75 \mathrm{keV}$ \\
$7 \mu \mathrm{m}$ & $87 \mathrm{keV}$ \\
$10 \mu \mathrm{m}$ & $95 \mathrm{keV}$ \\
$20 \mu \mathrm{m}$ & $120 \mathrm{keV}$ \\
$50 \mu \mathrm{m}$ & $180 \mathrm{keV}$ \\
$100 \mu \mathrm{m}$ & $260 \mathrm{keV}$ \\
\hline \multicolumn{2}{|c|}{ Diamond crystal [4] } \\
\hline $6 \mu \mathrm{m}$ & $77 \mathrm{keV}$ \\
$21 \mu \mathrm{m}$ & $116 \mathrm{keV}$ \\
$39 \mu \mathrm{m}$ & $153 \mathrm{keV}$ \\
$60 \mu \mathrm{m}$ & $195 \mathrm{keV}$ \\
\hline
\end{tabular}

During computations of a heat transfer within the potential configurations of the measuring head designed for the TORE SUPRA experiment it was found that from a point of view of the heat engineering it might be promising to replace the continuous Cherenkov measurement in a neighbourhood of bulk plasma by a sequence of short-lasting measurements, i.e., to introduce and withdraw the detector head from a plasma region [5]. It means also that such measurements can be carried out several times during a single tokamak relatively long-lasting discharge, and the Cherenkov detector might be introduced into a region of high electron fluxes for a short 
period (below 1 second) only. Solely the permanent assignment of probe could be applied for small and medium tokamaks (producing relatively low thermal loads).

The Cherenkov radiation, as generated inside the radiators, is usually lead out through separate fibres (optical cables) to the atmospheric pressure side. The emitted radiation in the blue (near ultraviolet) spectrum range should be collected and delivered through optical cables to a control room, then amplified (2-3) x $10^{7}$ times by appropriate photomultipliers and recorded with fast oscilloscopes.

\section{Practical applications}

The Cherenkov-type single-channel detector was for the first time applied in the CASTOR experiment in Prague [6-7]. The detection head (see Fig. 1) contained a radiator made of a small aluminium-nitride (AIN) crystal protected from the visible light by a Ti-layer of about $10 \mu \mathrm{m}$ in thickness [6]. The AlN crystal radiator was chosen due to its relatively low energy threshold, good thermal conductivity and a relatively low price. The observed difference between the Cherenkov- and $\mathrm{H}_{\alpha}$-signals confirmed that the detection head was well protected from the visible radiation. The obtained results showed that the Cherenkov measuring circuit might be influenced by intense hard X-rays. To eliminate the X-ray influence, some changes in the detector head construction were made [7] - the radiator and light-pipe was optically coupled by means of a metal tube, after elimination of coupling quartz rod.
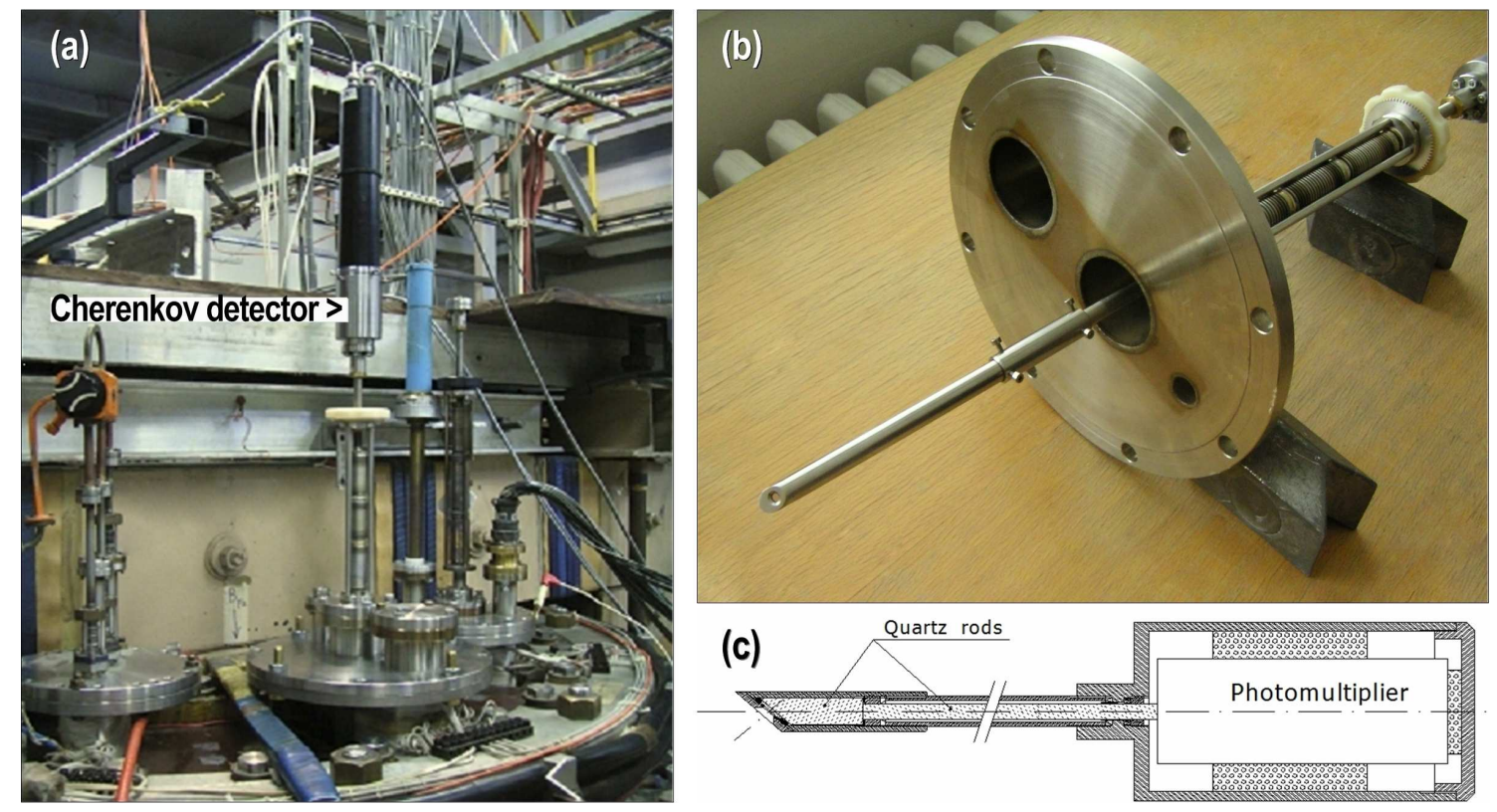

Fig. 1. Overview of the Cherenkov radiation detection system. (a) The measuring head, which was mounted upon the top diagnostic port of the CASTOR tokamak at IPP in Prague, (b) its general view and (c) its scheme [6].

On the basis of that experiment a four-channel Cherenkov probe with diamond crystal detectors was manufactured and applied later on within the TORE SUPRA facility [4, 8], as shown in Fig. 2. Several versions of measuring heads have been manufactured and used also within the ISTTOK tokamak [3, 9-10]; while only one single-channel measuring head was so far applied within the COMPASS device [11]. During recent experiments within the FTU tokamak 
in Frascati particular attention has been paid to measurements of runaway electrons, and a relationship between the Cherenkov detector signals and the magnetic island rotation has been observed [12].
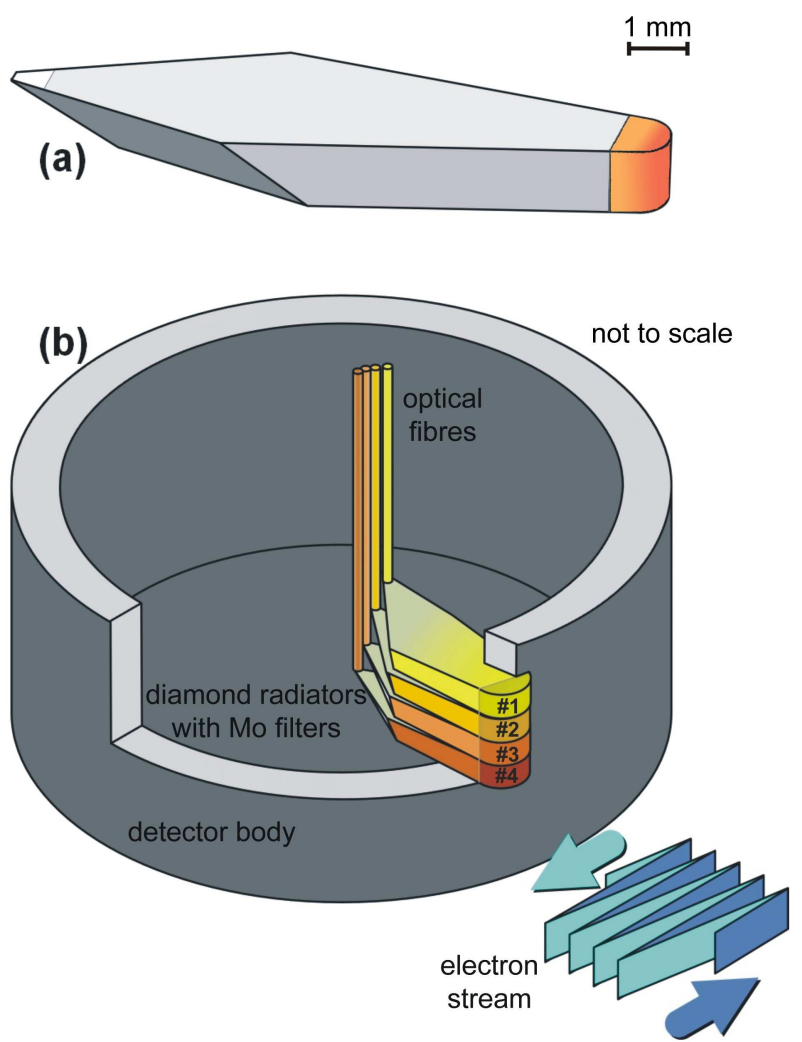

Fig. 2. General idea of the four-channel Cherenkov-type detector head construction for TORE SUPRA experiments [4]: (a) A single diamond with thin metal coatings deposited upon all the radiator surfaces, except for the part marked in orange. The front (rounded) corner was coated by the Mo-filter.; (b) Spatial arrangement of diamond radiators and optical fibres.

The overall view of detector four-channel detection head fixed on the manipulator of ISTTOK tokamak is presented in Fig. 3.

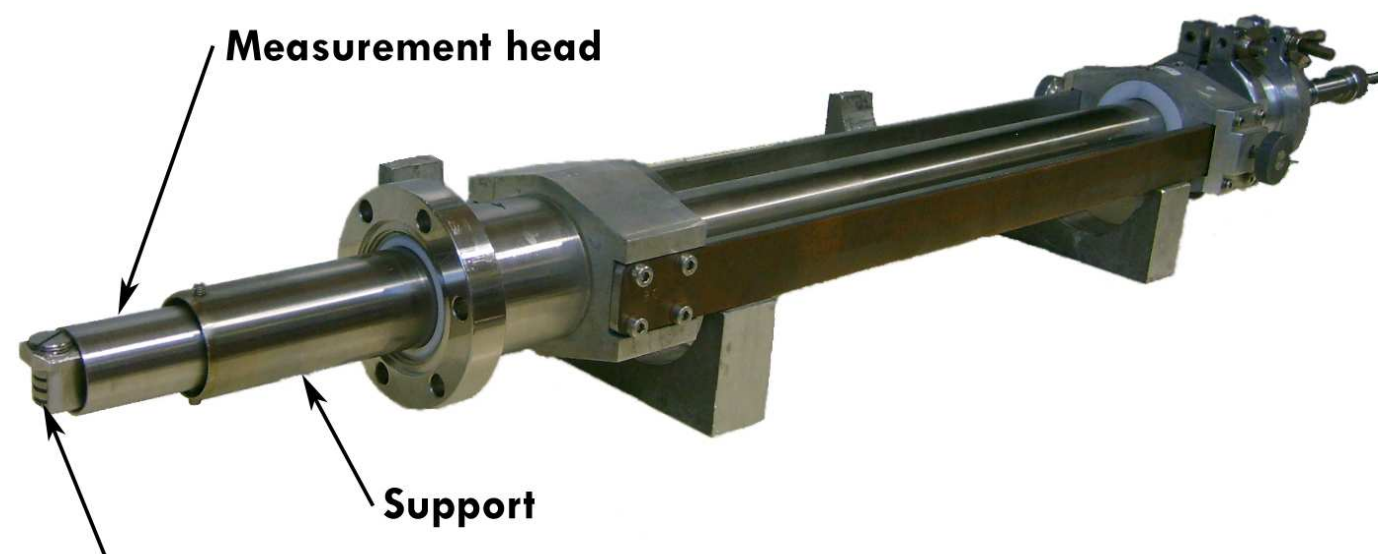

AIN crystal radiators with $\mathrm{Ti}$ - coating

Fig. 3. Four-channel Cherenkov-type detector head installed within the positioning equipment for ISTTOK experiments [3]. 


\section{Conclusions}

Several detectors exploiting the Cherenkov effect were successfully applied for the investigation of fast electron beams within various tokamak-type devices of different operational parameters.

The application of the Cherenkov-type diagnostics made it possible to measure run-away, ripple-born and suprathermal electrons from the main plasma discharge. The run-away electrons, which are generated when the friction of electron collisions with plasma does not compensate the externally induced electrical force, may reach energies up to several dozen $\mathrm{MeV}$. The ripple-born electrons, generated within imperfect toroidal field from limited number of magnetic coils, may be observed in the energy range from about $50 \mathrm{keV}$ to several hundreds $\mathrm{keV}$. Supratermal electrons could be measured at least in small and medium size devices.

It is possible to modify the Cherenkov detectors for specific experimental conditions of different tokamaks, expected electron beam parameters, as well as heat loads conditions. A comparison of the obtained signals with the experimental results from other diagnostics could significantly enlarge the knowledge of physical phenomena occurring in tokamak plasmas.

\section{Acknowledgements}

The reported studies were performed as the task of the research programme supported by the EURATOM Community under a contract with the Association EURATOM-IPPLM, Poland (Contract no. FU07-CT-2007-00061), by the Ministry of Science and Higher Education, Poland, under the contracts 1441/7.PR—EURATOM UE/2010/7, W51/7.PR—EURATOM/2011, 2498/7.PR EURATOM/2012/2 and 2861/7.PR EURATOM/2013/2.

\section{References}

[1] L. Jakubowski, M. Sadowski, J. Żebrowski; J. Tech. Phys. 38, 1, 141, 1997.

[2] M.J. Sadowski, L. Jakubowski, A. Szydłowski; Czech. J. Phys. 54, C74, 2004.

[3] L. Jakubowski, K. Malinowski, M.J. Sadowski, et al.; Nucl. Instr. Meth. A Vol. 623, 686, 2010.

[4] L. Jakubowski, M.J. Sadowski, J. Żebrowski, et al.; Rev. Sci. Instrum. Vol. 84, 016107, 2013.

[5] L. Jakubowski, M. Rabiński, et al.; Probl Atom Sci Tech 13:206-208, 2007.

[6] L. Jakubowski, J. Stanisławski, et al.; Czech. J. Phys. Vol. 56 No Suppl. B, B98, 2006.

[7] L. Jakubowski, M.J. Sadowski, J. Stanisławski, et al.; AIP Conf. Proc. Vol. 996, 219, 2008.

[8] L. Jakubowski, M.J. Sadowski, J. Żebrowski, et al.; Rev. Sci. Instrum. Vol. 81, 013504, 2010.

[9] V.V. Plyusnin, L. Jakubowski, et al.; Rev. Sci. Instrum. Vol. 83 No 8, 083505, 2012.

[10] L. Jakubowski, V.V. Plyusnin, et al.; Nucl. Instr. Meth. A Vol. 767, 61, 2014.

[11] L. Jakubowski, M.J. Sadowski, J. Żebrowski, et al.; Physica Scripta T161, 014011, 2014.

[12] F. Causa, et al.; 25 $5^{\text {th }}$ IAEA Fusion Energy Conf, St Petersburg, IAEA CN-221, p95, 2014. 